\title{
SOME PROPERTIES OF THE PROPER VALUES OF A MATRIX ${ }^{1}$
}

\author{
Y. K. WONG
}

Introduction. In [9], we consider a square matrix $A=\left(a_{i j}\right)$ with real or complex elements satisfying the inequalities

$$
s_{i}=\sum_{i=1}^{n}\left|a_{i j}\right| \leqq 1 \quad(j=1,2, \cdots, n) .
$$

Then $I-A$ is nonsingular if

$$
\left|a_{11}\right|<1, \quad\left|a_{k k}\right|+\sum_{i=1}^{k-1} s_{i}\left|a_{i k}\right|<1 \quad(k>1) .
$$

With property (1), condition (2) is implied by

$$
\sum_{i=1}^{j}\left|a_{i j}\right|<1 \quad(j=1,2, \cdots, n) .
$$

In [10], we show that if $A$ is non-negative and satisfies (1), then properties (2) and (3) are equivalent, and either property (2) or (3) is a necessary and sufficient condition for the maximal proper value to be less than unity. In this paper, we study some properties of the proper values of a matrix without condition (1). Without assuming (1), property (3) is necessary but not sufficient for a maximal proper value of a non-negative matrix to be less than 1 . With condition (1), property (2) is a sufficient condition but not necessary for a real- or complex-valued matrix to have a maximal proper value less than unity in modulus. In $\S 3$, we give some equivalent conditions for all the proper values of a non-negative matrix $A$ to be less than unity in modulus. Property (v) in $\S 3$ shows that $a_{k k}+c_{k}<1$ (see (3.4) and (3.5) below) is an equivalent condition for all the proper values to lie within a unit circle. This property is analogous to property (2) above. Another equivalent property is stated as follows: there exists a principal submatrix $A_{(p-1)}$ and a positive vector $w$ (both) of order $n-1$ such that $w^{\prime} A_{(p-1)}<w^{\prime}$ and $\operatorname{det}(I-A)>0$.

1. Moduli of finite matrices. The modulus of a finite matrix $A$ with real or complex elements is a finite real-valued function, $\|A\|$, satisfying the following axioms:

Presented to the Society, December 29, 1954; received by the editors May 16, 1953 and, in revised form, May 12, 1954.

1 The paper was prepared under Office of Naval Research Contract N6onr-27009. 
(I) $\|s A\|=|s| \cdot\|A\|$ for every number $s$.

(II) $\|I\|=1$, independent of the order of $I$.

(III) $\|A B\| \leqq\|A\| \cdot\|B\|$.

(IV) $\|A+B\| \leqq\|A\|+\|B\|$.

(V) For every submatrix $E$ of an identity matrix $I,\|E\| \leqq\|I\|$.

(VI) If $\lim A_{p}=A$, then $\lim _{p}\left\|A-A_{p}\right\|=0$.

(VII) If $A, B$ are two non-negative matrices, then $\|A+B\|$ $\geqq \max (\|A\|,\|B\|)$.

The first five axioms give the properties of "norms" in Banach algebras. Axiom (VI) is valid only for finite matrices. The assumption of non-negativeness in (VII) is essential. For if $A$ and $B$ are real or complex valued and $B=-A \neq 0$, then (VII) is not valid. Axiom (V) is equivalent to $\left(\mathrm{V}_{0}\right)$ : The modulus of a matrix is not less than the modulus of any one of its submatrices. Axiom (VII) may be replaced by the following statement: $\left(\mathrm{VII}_{0}\right)$ Let the matrices $A$ and $B$ be nonnegative such that $A \geqq B$ (i.e. each element of $A$ is at least equal to the corresponding element of $B$ ); then $\|A\| \geqq\|B\|$.

The following lemma depends on only (I), (II), (III), and (VI).

Lemma 1.2 The modulus of a maximum proper value of a finite square matrix $A$ with real or complex elements is equal to $\lim _{p}\left\|A^{p}\right\| 1 / p$.

Proof. The limit stated in the lemma exists and is finite. For, let $a_{p}=\log \left\|A^{p}\right\|$. Then (II) shows that $a_{0}=0$ and (III) shows that $a_{p+q} \leqq a_{p}+a_{q}$. Pólya and Szegö [5, p. 171, Problem 98] show that $\lim \left(a_{p} / p\right)=\inf \left(a_{p} / p\right)=b \geqq-\infty$. Then $\exp (b) \leqq\|A\|$, proving that the limit is finite.

Let $\lambda_{1}$ be a proper value with maximum modulus. Then $\lambda_{1}^{p} x=A^{p} x$, where $x$ is not a zero vector. We may assume that $\|x\|=1$. Hence by (I) and (III), we have $\left|\lambda_{1}\right|^{p} \leqq\left\|A^{p}\right\|$, and hence $\left|\lambda_{1}\right| \leqq\left\|A^{p}\right\|^{1 / p}$.

Let $r=\lim \left\|A^{p}\right\| 1 / p$. We shall show that $\left|\lambda_{1}\right|$ cannot be less than $r$. If $\left|\lambda_{1}\right|<r$, let $s$ be such that $\left|\lambda_{1}\right|<s<r$. Then the Carl Neumann's series $R(s, A)=s^{-1} \sum_{0}^{\infty} s^{-p} A^{p}$ converges. It follows that $s^{-p} A^{p}$ converges to zero as $p$ tends to infinity. By the continuity property of the modulus function (see Axiom (VI)), for sufficiently large $p$, $\left\|s^{-p} A^{p}\right\|<1$, and hence $\left\|A^{p}\right\| 1^{1 / p}<s$ for sufficiently large $p$. This result is absurd, as $s<r$.

For future development, we mention two instances. Consider realor complex-valued matrices and vectors. Let $A^{*}$ denote the conjugate-transpose of $A$ with $m$ rows and $n$ columns. Thus $x^{*} x$ gives a non-negative number. We introduce

2 A generalized result in terms of spectral norm in Banach algebra is known. 


$$
\begin{gathered}
\left\|x^{*}\right\|_{1}=\|x\|_{1}=\left(\sum_{i}\left|x_{i}\right|^{2}\right)^{1 / 2} \\
\left\|x^{*}\right\|_{2}=\max \left(\left|x_{1}\right|, \cdot,\left|x_{m}\right|\right), \quad\|x\|_{2}=\sum_{i}\left|x_{i}\right| .
\end{gathered}
$$

Define for $\nu=1,2$,

$$
\|A\|_{\nu}=\sup \left\{\left|x^{*} A y\right|\|\| x^{*}\left\|_{\nu}=\right\| y \|_{\nu}=1\right\} .
$$

Then

$$
\|A\|_{1}=\left\|A^{*}\right\|_{1} ; \quad\left\|A^{*} A\right\|_{1}=\left\|A A^{*}\right\|_{1}=\|A\|_{1}^{2}=\lambda,
$$

where $\lambda$ is a maximum proper value of $A^{*} A$. See [4, pp. 1042-1044];

$$
\|A\|_{2}=\max _{k}\left\{\sum_{i}\left|a_{j k}\right|\right\} \text {. }
$$

Let us prove (1.5). We can verify that

$$
\left|x^{*} A y\right| \leqq\left\|x^{*}\right\|_{2}\|A\|_{2}\|y\|_{2}=\|A\|_{2}
$$

if the moduli of $x^{*}$ and $y$ are equal to 1 . On the other hand, suppose that the $r$ th column gives the maximum value. Let $\bar{a}_{j r}$ be the conjugate of $a_{j r}$. Take $x^{*}=\left(\bar{a}_{j r} /\left|a_{j r}\right| \mid j=1, \cdots, m\right)$, and $y=\delta_{r}$, the $r$ th column of the identity matrix of order $n$. Then $\left\|x^{*}\right\|_{2}=\|y\|_{2}=1$ and $\left|x^{*} A y\right|=\sum_{j}\left|a_{j r}\right|$, which together with (1.6) gives (1.5).

One can verify that (1.4) or (1.5) satisfies Axioms (I) to (VII). To prove the continuity property (VI) of $\|A\|_{1}$, we make use of $\|A\|_{1}$ $\leqq\left(\sum_{i, j}\left|a_{i j}\right|^{2}\right)^{1 / 2}$. If $A$ consists of non-negative numbers, then $x, y$ may be restricted to be non-negative in (1.3) without altering the modulus of $A$. This fact is used to prove (VII). The results in $\S 1$ hold for real quaternions.

2. Finite square matrices and their proper values. In the sequel, we shall let $\lambda_{1}$ be a proper value of $A$ with maximum modulus.

TheOREM 1. Let $A=\left(a_{i j}\right)$ be a finite square matrix of order $n$ with non-negative elements. If $\lambda_{1}<1$, then by permutations of rows and columns, $A$ has the property

$$
\sum_{i=1}^{k} a_{i k}<1, \quad k=1,2, \cdots, n .
$$

$A$ similar property holds for the rows of $A$.

Proof. Let $s_{k}=\sum_{i=1}^{n} a_{i k}$ for $k=1,2, \cdots, n$. The minimum of all $s_{k}$ is less than 1 . For, if $\min _{k}\left(s_{k}\right) \geqq 1$, then $\lambda_{1} \geqq 1$. Thus $\lambda_{1}<1$ implies that there exists at least one column-sum, say $s_{n}$, less than unity. From Axiom (VII) or (VII $)_{0}$, one can deduce that if $B$ is a non- 
negative matrix whose elements are not greater than the corresponding ones of $A$, then (by Lemma 1), the maximum proper value of $B$ is not greater than that of $A$. Hence, the maximum proper value of any principal submatrix of $A$ is not greater than that of $A$. Let $A_{k}$ be the principal submatrix consisting of the first $k$ rows and columns of $A$. Then there exists in $A_{k}$ a column-sum less than unity. Let such a column be the $k$ th one. This proves (2.1).

Note that condition (2.1) is not sufficient for $\lambda_{1}<1$ as counterexamples show. Property (2.1) is not valid if we merely assume $A$ to be real-valued, for, in our proof, Axiom (VII) is used.

If we assume that all $s_{k}$ are at most unity, then (2.1) is sufficient for $\lambda_{1}<1$. But this condition is even valid for real or complex valued matrices.

TheOREM 2. Let $A$ be a finite square matrix of order $n$ with real or complex elements such that $s_{k}=\sum_{i=1}^{n}\left|a_{i k}\right| \leqq 1$ for all $k$. Then

$$
\sum_{i=k}^{n}\left|a_{i k}\right|<1, \quad k=1,2, \cdots, n,
$$

implies

$$
\left|a_{k k}\right|+\sum_{j=k+1}^{n} s_{j}\left|a_{j k}\right|<1, \quad k=1, \cdots, n-1 ;\left|a_{n n}\right|<1 .
$$

Property (2.3) implies the existence of an integer $p \leqq n$ such that $\left\|A_{p}\right\|_{2}<1$.

$\|A\|_{2}$ is defined by (1.5). The $i$ th row and $j$ th column of $A$ are denoted by $A(i, \cdot)$ and $A(\cdot, j)$ respectively. $A(i, j)$ and $a_{i j}$ have the same meaning.

Proof. We can see easily that (2.2) implies (2.3). By (2.3), we have $\|A(\cdot, 1)\|_{2}<1$. Let $c_{j}=\left\|A^{q}(\cdot, j)\right\|_{2}$, where $0<q<n$. Suppose that $c_{r}<1$ for $r=1,2, \cdots, q$. Then for $k=1,2, \cdots, q$,

$$
\left\|A^{q+1}(\cdot, k)\right\|_{2} \leqq\|A\|_{2}\left\|A^{q}(\cdot, k)\right\|_{2} \leqq 1 \cdot c_{k}<1 .
$$

Let $k=q+1$ or larger; then

$$
\begin{aligned}
\sum_{i=1}^{n}\left|A^{q+1}(i, k)\right| & =\sum_{i=1}^{n}\left|A^{q}(i, \cdot) A(\cdot, k)\right| \\
& \leqq \sum_{i=1}^{n}\left(\sum_{i=1}^{n}\left|A^{q}(i, j)\right| \cdot|A(j, k)|\right) \\
& =\sum_{r=1}^{q} c_{r}\left|a_{r k}\right|+\sum_{t>q} c_{t}\left|a_{t k}\right| .
\end{aligned}
$$


Now we have two cases. (i) $a_{1 k}=\cdots=a_{q k}=0$; then the preceding expression is

$$
=\sum_{t>q} c_{t}\left|a_{t k}\right| \leqq \sum_{t=q+2}^{n} s_{t}\left|a_{t k}\right|+\left|a_{q+1, k}\right|<1 .
$$

(ii) $a_{r k} \neq 0$ for some $r(=1, \cdots, q)$; as $c_{r}<1$ the last expression in (2.4) is

$$
<\sum_{r=1}^{q}\left|a_{r k}\right|+\sum_{t>q} c_{t}\left|a_{t k}\right| \leqq \sum_{i=1}^{n}\left|a_{i k}\right|=s_{k} \leqq 1 .
$$

In either case, we have $\left\|A^{q+1}(\cdot, k)\right\|_{2}<1$ for $k=q+1$ at least. Thus our theorem is proved.

The important fact is the smallness of the integer $p$. If conditions $s_{k} \leqq 1$ for all $k$ and (2.3) are not assumed, then the integer $p$ is usually very large, unless $A$ is nilpotent. The preceding theorem is valid for real quaternions.

LEMMA 2. If there exist an integer $p$ and a non-negative number $c$ such that $\left\|A^{p}\right\| \leqq c<1$, then $\lim _{q}\left\|A^{q}\right\| 1 / q<1$ and conversely.

The lemma is well known in Banach algebras. (See the proof of Lemma 1.)

Corollary 1. If condition (2.2) or (2.3) is satisfied, then $\left|\lambda_{1}\right|<1$.

Lemma 3. Let $A$ be real-or complex-valued and indecomposable such that $s_{k} \leqq 1$ for $k>1$ and $s_{1}<1$. Then condition (2.2) holds, subject to the permutations of rows and columns.

Proof. Condition (2.2) is satisfied for $k=1$. Let $k=2, \cdots, n$. Consider the submatrix $\left(a_{p q}\right)$ for $p=1, \cdots, k-1, q=k, \cdots, n$. The indecomposability shows that there is at least one element different from zero. If one of the elements $a_{1 k}, a_{2 k}, \cdots, a_{k-1, k}$ is different from zero, then

$$
\sum_{i=k}^{n}\left|a_{i k}\right|<s_{k} \leqq 1 .
$$

If any other element is different from zero, we make the necessary interchange of rows and columns to achieve the desired result. (Lemma 3 holds also for real quaternions.)

By Lemma 3 and Corollary 1 , we get the following result of A. T. Brauer [1, pp. 876-877].

Corollary 2. Let $A$ have the properties in the hypothesis of Lemma 3. Then all the proper values of $A$ are less than 1 in modulus. 
3. Non-negative matrices. In this section we give some equivalent conditions for $\lambda_{1}<1$. Not all the conditions are new; for example, (i) is due to Frobenius [2] and (ii) is due to Carl Neumann [3]. However, our proof is elementary and simple. We shall assume $A$ to be non-negative-valued.

Lemma 4. If $(I-A)^{-1}$ exists and has non-negative values, then $a_{k k}<1$ for all $k$, and the diagonal elements of $(I-A)^{-1}$ are at least equal to unity. ${ }^{3}$

Proof. Let $\left(r_{i j}\right), i, j=1, \cdots, n$, be the inverse of $I-A$. The inner product of the $k$ th row of $\left(r_{i j}\right)$ and the $k$ th column of $I-A$ gives

$$
r_{k k}\left(1-a_{k k}\right)-\sum_{h \neq k} r_{k h} a_{h k}=1 \text {. }
$$

If $1-a_{k k} \leqq 0$, the left-hand side of (3.1) would be nonpositive, which is impossible. Thus $1-a_{k k}>0$. From (3.1), it follows that $r_{k k}\left(1-a_{k k}\right)$ $\geqq 1$. As $0<1-a_{k k} \leqq 1$, we have $r_{k k} \geqq 1$.

The following properties are mutually equivalent.

(i) The inverse of $I-A$ exists and has non-negative values.

(ii) The series $I+A+A^{2}+\cdots$ converges.

(iii) Every principal submatrix $B$ of $A$ has the property that the inverse of $I-B$ exists and is non-negative.

Before stating property (iv), we adopt some notations. For $p$ ranging from 1 to $n$, let $A_{p}$ be the principal submatrix consisting of the elements in the first $p$ rows and columns of $A ; A_{-p}$ the principal submatrix of order $n-p$ omitting all the elements in the first $p$ rows and columns; $C=\left(a_{i r}\right), D=\left(a_{s j}\right)$ with $i, j=1, \cdots, p$ and $r, s=p^{+1}$, $\cdots, n$. We express $I-A$ in the form

$$
I-A=\left(\begin{array}{cc}
L & -C \\
-D & M
\end{array}\right)=\left(\begin{array}{cc}
I & 0 \\
-D L^{-1} & I
\end{array}\right)\left(\begin{array}{cc}
L & 0 \\
0 & K_{p}
\end{array}\right)\left(\begin{array}{cc}
I & -L^{-1} C \\
0 & I
\end{array}\right)
$$

where $L=I-A_{p}, M=I-A_{-p}$, and

$$
K_{p}=I-A_{-p}-D\left(I-A_{p}\right)^{-1} C .
$$

(iv) For each $p=1, \cdots, n-1$, the inverses of $I-A_{p}$ and $K_{p}$ exist and are non-negative.

(v) For each $p=1,2, \cdots, n-1$, the inverse of $I-A_{p}$ exists and is non-negative, and for $k=1,2, \cdots, n$,

$$
c_{k}<1-a_{k k}
$$

where

${ }^{3}$ A related result was obtained by J. H. Curtiss in "Monte Carlo" methods for the iteration of linear operators, Journal of Mathematics and Physics vol. 32 (1954) p. 224. 


$$
c_{1}=0, \quad c_{k}=\sum_{r, s=1}^{k-1} a_{k r}\left(I-A_{k-1}\right)^{-1}(r, s) a_{s k} .
$$

Similar results hold for every permutation on $1, \cdots, n$.

(vi) There exists a sequence of principal submatrices $A_{(p)}, p$ $=1, \cdots, n$, such that (1) $A_{(p)}$ of order $p$ is a principal submatrix of $A_{(p+1)}$, and (2) $d_{p+1} \leqq d_{p}$ where $d_{p}=\operatorname{det}\left(I-A_{(p)}\right)$. Moreover, $d_{n}>0$.

(vii) There exists a sequence of principal submatrices $A_{(p)}$, $p=1, \cdots, n$, such that (1) $A_{(p)}$ of order $p$ is a principal submatrix of $A_{(p+1)}$, and (2) $\operatorname{det}\left(I-A_{(p)}\right)>0$ for $p=1, \cdots, n$.

(viii) To each vector $y$ with non-negative components, the equation $x^{\prime}(I-A)=y^{\prime}$ has a solution such that $x \geqq y$. Moreover, if $y_{k}>0$ for some $k$, then $x_{k}>0$.

(ix) A vector $z$ with positive components exists such that $z^{\prime} A<z^{\prime}$.

(x) The maximum proper value $\lambda_{1}$ of $A$ is less than unity.

Proof. We shall show that the first seven properties imply each other in cyclic order. (i) implies (ii): By Lemma 4, we write $(I-A)^{-1}$ $=I+A_{*}$; then $A_{*}$ has non-negative values and commutes with $A$. For $m \geqq 1$,

$$
A_{*}=A+A^{2}+\cdots+A^{m}+A^{m} A_{*} .
$$

Let $S_{m}=I+A+\cdots+A^{m}$. Then $S_{m} \leqq S_{m+1}$ and every element of $S_{m}$ is bounded above by the corresponding element of $(I-A)^{-1}$. Hence $\lim _{m} S_{m}$ exists and is the inverse of $I-A$. That (ii) implies (iii) is obvious. (iii) implies (iv): From (iii), it follows that the inverse of $I-A_{p}$ exists and is non-negative. That the inverse of $K_{p}$ exists and is non-negative follows from (3.2) and (iii). (iv) implies (v): Let $L=I$ $-A_{p}$ for $p=1,2, \cdots, n-1$ in (3.2). Then (iv) states that the inverse of $I-A_{p}$ exists and is non-negative. Obviously (3.4) holds for $k=1$. Let $p=2, \cdots, n$, and apply (3.2) to $I-A_{p}$ with $L=I-A_{p-1}$. Then $K_{p}$ becomes $1-a_{p p}-c_{p}$. Since the inverse of $I-A_{p}$ exists and is nonnegative, it follows that $1-a_{p p}-c_{p} \neq 0$ and is non-negative, i.e. positive. (v) implies (vi): Let $A_{(p)}=A_{p}$ as specified above. Then property (1) in (vi) is satisfied. We can show $[8$, p. 234] that for $p=2, \cdots, n$, $d_{p}=d_{p-1}\left(1-a_{p p}-c_{p}\right)$, which by (v), proves property (2) in (vi) and also $d_{n}>0$. That (vi) implies (vii) is evident. (vii) implies (i): Since $d_{n}>0$, the inverse of $I-A$ exists. To prove the non-negativeness of $(I-A)^{-1}$, we reduce $I-A$ into a diagonal matrix by a method similar to (3.2). Let $e_{1}=d_{1}, e_{p}=d_{p} / d_{p-1}$ for $p>1$. By hypothesis, $e_{1}, \cdots, e_{n}$ are positive. Put $B_{1}=A$. From the non-negative matrix $B_{p}$ of order $n-p+1$ such that the first element in the diagonal of $I-B_{p}$ is $e_{p}$, we construct $B_{p+1}$ of order $n-p$ by the method of (3.2) as follows: Let $B_{p}^{0}$ be the principal submatrix of $B_{p}$ with the first row and 
column omitted; $\beta_{p}$ and $\alpha_{p}^{\prime}$ be the first column and row respectively of $B_{p}$ with the first element omitted. Define $B_{p+1}=B_{p}^{0}+\beta_{p} e_{p}^{-1} \alpha_{p}^{\prime}$. Then $B_{p+1}$ is non-negative, and

$$
I-B_{p}=\left(\begin{array}{cc}
1 & 0 \\
-\beta_{p} e_{p}^{-1} I
\end{array}\right)\left(\begin{array}{cc}
e_{p} & 0 \\
0 & I-B_{p+1}
\end{array}\right)\left(\begin{array}{cc}
1 & -e_{p}^{-1} \alpha_{p}^{\prime} \\
0 & I
\end{array}\right) .
$$

By the elementary properties of determinants, the first element in the diagonal of $I-B_{p+1}$ is $e_{p+1}$. Thus, $I-A=(I-P) E(I-Q)$, where $E$ is a diagonal matrix with $e_{1}, \cdots, e_{n}$ in its diagonal, $P$ and $Q$ have non-negative elements respectively below and above the principal diagonal, and zeros elsewhere. Hence $(I-A)^{-1}=(I+Q) E^{-1}(I+P)$, which is non-negative. This completes the proof that the first 7 properties are equivalent to one another.

To complete the proof, we shall show that (i), (viii), (ix), (x), and (iii) imply one another in that order. That (i) implies (viii) follows from Lemma 4. (viii) implies (ix): For a positive $y$, we have, from (viii), a positive $u$ such that $u^{\prime}-u^{\prime} A=y^{\prime}>0$. (ix) implies (x): If $A$ is nilpotent, then $\lambda_{1}=0<1$. If $A$ is not nilpotent, by Frobenius' result [2], $\lambda_{1}>0$ and $A v=\lambda_{1} v$ where $v$ is non-negative. Hence $\lambda_{1} u^{\prime} v$ $=u^{\prime} A v<u^{\prime} v$. Since $u^{\prime} v>0$, we have property (x). That property (x) implies (iii) is a well known result concerning the Carl Neumann's series [7, pp. 18-19].

Note that the weak condition (vii) implies that all the principal minors of $I-A$ are positive. The following equivalent condition is useful in practical applications:

Corollary. The maximal proper value $\lambda_{1}$ of $A$ is less than 1 if and only if there exist a principal submatrix $A_{(n-1)}$ and a positive vector $w$ (both) of order $n-1$ such that $w^{\prime} A_{(n-1)}<w^{\prime}$ and $\operatorname{det}(I-A)>0$.

Added in the proof. There is redundance in (v). The property given by (3.4) and (3.5) implies the existence of non-negative inverses of $I-A_{p}$ for all $p$. The proof is by induction. See the demonstration for which (vii) implies (i).

\section{REFERENCES}

1. A. T. Brauer, Limits for the characteristic roots of a matrix, III, Duke Math. J. vol. 15 (1948) pp. 871-877.

2. G. Frobenius, Über Matrizen aus nicht-negativen Elementen, K. Preuss. Akad. Sitzungsber. (1912) pp. 456-477.

3. C. Neumann, Untersuchungen über das logarithmische und Newtonsche Potential, Leipzig, Teubner, 1877, XVI, p. 368.

4. J. von Neumann and $\mathrm{H}$. Goldstine, Inverting matrices of higher order, Bull. Amer. Math. Soc. vol. 53 (1947) pp. 1021-1099. 
5. G. P6lya and G. Szegö, Aufgaben und Lehrsatze aus der Analysis, I, Berlin, Springer, 1925.

6. G. B. Price, Bounds for determinants with dominant principal diagonal, Proc. Amer. Math. Soc. vol. 2 (1951) pp. 497-502.

7. A. Wintner, Spektraltheorie der unendlichen Matrizen, von S. Hirzel, 1929.

8. Y. K. Wong, Some inequalities of determinants of Minkowski type, Duke Math. J. vol. 19 (1952) pp. 231-241.

9. ——, An inequality for Minkowski matrices, Proc. Amer. Math. Soc. vol. 4 (1953) pp. 139-141.

10. - On nonnegative valued matrices, Proc. Nat. Acad. Sci. U.S.A. vol. 40 (1954) pp. 121-124.

11. J. H. Curtiss, "Monte Carlo" methods for the iteration of linear operators, Journal of Mathematics and Physics vol. 32 (1954) pp. 209-232.

Princeton University

\section{PROJECTIONS IN THE SPACE $(m)^{1}$}

\section{ROBERT C. JAMES}

A projection in a Banach space is a continuous linear mapping $P$ of the space into itself which is such that $P^{2}=P$. Two closed linear manifolds $M$ and $N$ of a Banach space $B$ are said to be complementary if each $z$ of $B$ is uniquely representable as $x+y$, where $x$ is in $M$ and $y$ in $N$. This is equivalent to the existence of a projection for which $M$ and $N$ are the range and null space [7, p. 138]. It is therefore also true that closed linear subsets $M$ and $N$ of $B$ are complementary if and only if the linear span of $M$ and $N$ is dense in $B$ and there is a number $\epsilon>0$ such that $\|x+y\| \geqq \epsilon\|x\|$ if $x$ is in $M$ and $y$ in $N$.

It is known that a Banach space $M$ is complemented in each Banach space in which it can be embedded if it is isomorphic with a complemented subspace of the space $(m)$ of bounded sequences. In particular, if $M$ is a subspace of a Banach space $Z$ and is isometric with a subspace $M^{\prime}$ of $(m)$, then there is a projection of $Z$ onto $M$ of norm less than or equal to $\lambda$ if there is a projection of $(m)$ onto $M^{\prime}$ of norm equal to $\lambda$ (see $[8$, p. 538] and $[9$, p. 945]). Thus the existence of a complement in $(m)$ for a subspace $M$ of $(m)$ is independent of the method by which $M$ is embedded in $(m)$. Any separable Banach space is isometric with a subspace of $(m)[3$, p. 107]. Hence a separable Banach space is complemented in each space in which it can be em-

\footnotetext{
Presented to the Society, October 30, 1954; received by the editors February 4, 1955.

1 Research supported in part by a grant from the National Science Foundation.
} 